
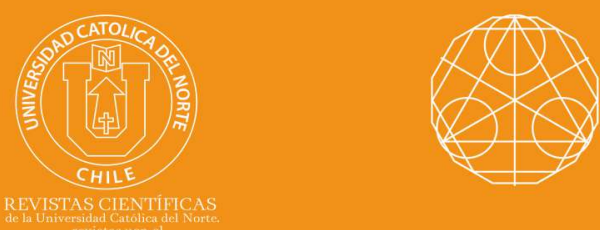

d oi" 10.22199/issn.0717-6279-4497 PROYECCIONES Journal of Mathematics

\title{
Equitably strong non-split equitable domination in graphs
}

\section{P. Nataraj ${ }^{1}$ (1) orcid.org/0000-0002-5535-7838}

R. Sundareswaran² orcid.org/0000-0002-0439-695X

V. Swaminathan ${ }^{3}$ (1) orcid.org/0000-0002-5840-2040

${ }^{1}$ The Madura College, Dept. of Mathematics, Madurai, TN, India.

natsssac7@yahoo.com

${ }^{2}$ Sri Sivasubramaniya Nadar College of Engineering, Dept. of Mathematics, Chennai, TN, India. sundareswaranr@ssn.edu.in

${ }^{3}$ Saraswathi Narayanan College, Ramanujan Research Center in Mathematics, Madurai, TN, India.

swaminathan.sulanesri@gmail.com

\section{Abstract:}

In a simple, finite and undirected graph $G$ with vertex set $V$ and edge set $E$, Prof. Sampathkumar defined degree equitability among vertices of $G$. Two vertices $u$ and $v$ are said to be degree equitable if $|\operatorname{deg}(u)-\operatorname{deg}(v)| \leq 1$. Equitable domination has been defined and studied in [7]. V.R. Kulli and B. Janakiram defined strong non - split domination in a graph [5]. In this paper, the equitable version of this new type of domination is studied.

Keywords: Equitable domination; Split domination; Strong non-split domination.

MSC (2020): 05C69.

\section{Cite this article as (IEEE citation style):}

P. Nataraj, R. Sundareswaran, and V. Swaminathan, "Equitably strong non-split equitable domination in graphs", Proyecciones (Antofagasta, On line), vol. 40, no. 4, pp. 989-999, 2021, doi: 10.22199/issn.07176279-4497

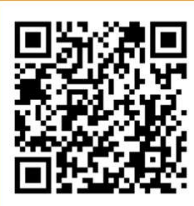

Article copyright: (C 2021 P. Nataraj, R. Sundareswaran and V. Swaminathan. This is an open access article distributed under the terms of the Creative Commons License, which permits unrestricted use and distribution provided the original author and source are credited.

(cc) BY 


\section{Introduction}

In 1973, W.Meyer defined equitable colouring [15] as a proper colouring in which the cardinalities of any two colour classes differ by at most one. Observing this, Prof. Sampathkumar defined degree equitability. Several papers were published using this concept namely equitable domination, equitable packing, equitably independent set, equitable vertex covering etc. $[1,2,3,7]$. In this paper, equitably complete subgraph is defined and equitable dominating set whose complement is an equitably complete subgraph is studied. Strong non-split domination was introduced by V.R.Kulli and B.Janakiram [4] wherein the foundation for the study of dominating sets whose complements induce complete subgraphs has been laid. The results of this paper are tried in the equitable context.

\section{Main results}

Definition 1. [1] Let $G$ be a simple graph with vertex $V(G)$ and edge set $E(G)$. Two vertices $u, v$ are said to be degree equitable if $|\operatorname{deg}(u)-\operatorname{deg}(v)| \leq$ 1. A subset $D$ of $V(G)$ is called an equitable dominating set of $G$ if for every $u$ in $V(G)-D$, there exists a $v \in D$ such that $u$ and $v$ are adjacent and degree equitable.

Definition 2. Let $D$ be an equitable domination set of $G$. $D$ is called an equitably strong non-split equitable dominating set if $\langle V-D\rangle$ is equitably complete. (That is, any two vertices of $G$ are adjacent and all the vertices of $V-D$ are degree equitable in $G$ ). The minimum(maximum) cardinality of a minimal strong non-split equitable dominating set of $G$ is called the strong non-split equitable domination number of $G$ (upper strong non-split equitable domination number of $G$ ) and is denoted by $\gamma_{e}^{s n s}(G)\left(\Gamma_{e}^{s n s}(G)\right)$.

If $G$ is not equitably totally disconnected, then there exists a vertex $u$ in $V(G)$ such that $V-\{u\}$ is a strong non-split equitable dominating set of $G$.

So, it is assumed that the graph that is considered is not equitably totally disconnected.

Observation 1. $\gamma_{e}(G) \leq \gamma_{e}^{n s}(G) \leq \gamma_{e}^{s n s}(G)$.

Theorem 1. A strong non-split equitable dominating set $D$ is minimal if and only if for every $u \in D$ one of the following conditions holds. 
1. $u$ is an equitable isolate $\langle D\rangle$.

2. $N_{e}(v) \cap D=\{u\}$ for some $v$ in $V-D$.

3. $u$ is not equitably adjacent with some vertex of $V-D$.

Proof. Obvious.

Proposition 1. $\beta_{e}(G) \leq \gamma_{e}^{\text {sns }}(G)$.

Proof. Let $D$ be a $\gamma_{e}^{\text {sns }}(G)$ - set of $G$. Let $T$ be an equitably independent set of $G$. Then $|T \cap(V-D)| \leq 1$. That is, either $T$ is a subset of $D$ or $T$ contains at most one vertex from $V-D$ and the remaining elements are from $D$. If $T$ contains a vertex say $v$ from $V-D$, then $T$ cannot contain the vertices in $D$ which are equitably adjacent with $v$. Since at least one vertex of $D$ is equitably adjacent with $v, T$ can contain at most $|D|-1$ vertices from $D$.

Remark 1. When $G=K_{n}, \beta_{e}(G)=\gamma_{e}^{\text {sns }}(G)=1$.

Definition 3. A subset $S$ of $V(G)$ is called an equitable clique if $\langle S\rangle$ is complete and all vertices of $S$ are degree equitable in $G$. The maximum cardinality of an equitable clique of $G$ is called the equitable clique number of $G$ and is denoted by $\omega_{e}(G)$.

Example 1. Let $G$ be obtained from $K_{4}$ by attaching two pendent vertices at exactly one vertex of $K_{4}$. Then $\omega(G)=4$ and $\omega_{e}(G)=3$.

Remark 2. $\omega_{e}(G) \leq \omega(G)$.

Theorem 2. $n-\omega_{e}(G) \leq \gamma_{e}^{\text {sns }}(G) \leq n-\omega_{e}(G)+1$.

Proof. Let $D$ be a $\gamma_{e}^{\text {sns }}(G)$ - set of $G$. Then $\langle V-D\rangle$ is an equitably complete subgraph of $G$. Therefore, $|\langle V-D\rangle| \leq \omega_{e}(G)$. That is, $n-\gamma_{e}^{\text {sns }}(G) \leq \omega_{e}(G)$ which gives $n-\omega_{e}(G) \leq \gamma_{e}^{s n s}(G)$.

Let $S$ be a $\omega_{e}(G)$ - subset of $G$. Then for any $u \in S, S-\{u\}$ is an equitable clique of $\mathrm{G}$. Since $\mathrm{u}$ is equitably adjacent with every element of $S-\{u\},(V-S) \cup\{u\}$ is a strong dominating set whose complement is equitably complete. Therefore, $\gamma_{e}^{\text {sns }}(G) \leq|(V-S) \cup\{u\}|=n-\omega_{e}(G)+1$. 
Remark 3. Let $G_{1}$ be the graph obtained from $K_{3}$ by attaching one pendent vertex at exactly two of the vertices of $K_{3}$. Then $\omega_{e}\left(G_{1}\right)=3$ and $\gamma_{e}^{\text {sns }}\left(G_{1}\right)=3$. Therefore, $\left|V\left(G_{1}\right)\right|-\omega_{e}\left(G_{1}\right)=5-3=2<\gamma_{e}^{\text {sns }}\left(G_{1}\right)=3$. Let $G_{2}=K_{3} \circ K_{1}$. Then $\omega_{e}\left(G_{2}\right)=3$ and $\gamma_{e}^{\text {sns }}\left(G_{2}\right)=4=n-\omega_{e}\left(G_{2}\right)+1$. Let $G_{3}$ be the graph obtained from $C_{4}$ by attaching a pendent vertex at exactly one vertex of $C_{4}$. $\left|V\left(G_{3}\right)\right|=5, \omega_{e}\left(G_{3}\right)=2$ and $\gamma_{e}^{\text {sns }}\left(G_{3}\right)=3$. Therefore, $n-\omega_{e}\left(G_{3}\right)=\gamma_{e}^{\text {sns }}\left(G_{3}\right)$. Let $G_{4}$ be obtained by attaching a single pendent vertex at exactly one vertex of $K_{3}$. Then $n=4, \omega_{e}\left(G_{4}\right)=3$ and $\gamma_{e}^{\text {sns }}\left(G_{4}\right)=2$. Therefore, $\gamma_{e}^{\text {sns }}\left(G_{4}\right)=2=n-\omega_{e}\left(G_{4}\right)+1$.

Theorem 3. Let $G$ be a graph with $\omega_{e}(G) \geq \delta_{e}(G)$. Then, $\gamma_{e}^{\text {sns }}(G) \leq$ $n-\delta_{e}(G)$.

Proof. Suppose $\omega_{e}(G) \geq \delta_{e}(G)+1$. Then $-\omega_{e}(G) \leq-\delta_{e}(G)-1$. From the above theorem, $\gamma_{e}^{\text {sns }}(G) \leq n-\omega_{e}(G)+1 \leq n-\delta_{e}(G)$. Suppose $\omega_{e}(G)=$ $\delta_{e}(G)$. Let $S$ be a $\omega_{e}(G)$-set of G. Let $u \in S$. Then, $\operatorname{deg}_{e}(u) \geq \delta_{e}(G)$. Since, $|S-\{u\}|<\delta_{e}(G), u$ is equitably adjacent with at least one vertex of $V-S$. Therefore, $V-S$ is an equitably strong non-split equitable dominating set of $\mathrm{G}$. Therefore, $\gamma_{e}^{\text {sns }}(G) \leq|V-S|=n-|S|=n-\delta_{e}(G)$.

Corollary 1. The above bounds are attained if and only if one of the following conditions is satisfied.

1. $\omega_{e}(G)=\delta_{e}(G)$.

2. $\omega_{e}(G)=\delta_{e}(G)+1$ and every $\omega_{e}(G)$ - set $S$ contains a vertex which is not equitably adjacent with any vertex of $V-S$.

1. Suppose $\omega_{e}(G)=\delta_{e}(G)$. Since $n-\omega_{e}(G) \leq \gamma_{e}^{\text {sns }}(G)$, we have $n-$ $\delta_{e}(G) \leq \gamma_{e}^{\text {sns }}(G)$. But from the above, $\gamma_{e}^{\text {sns }}(G) \leq n-\delta_{e}(G)$. Hence, $\gamma_{e}^{\text {sns }}(G)=n-\delta_{e}(G)$. Conversely, suppose, $\gamma_{e}^{\text {sns }}(G)=n-\delta_{e}(G)$. Then $n-\delta_{e}(G) \geq n-\omega_{e}(G)$. Therefore, $\omega_{e}(G) \geq \delta_{e}(G)$. Since, $\gamma_{e}^{s n s}(G) \leq n-\omega_{e}(G)+1, n-\delta_{e}(G)=\gamma_{e}^{s n s}(G) \leq n-\omega_{e}(G)+1$. That is, $\omega_{e}(G) \leq \delta_{e}(G)+1$. Therefore, $\omega_{e}(G)=\delta_{e}(G)$.

2. Suppose, $\gamma_{e}^{\text {sns }}(G)=n-\delta_{e}(G)$. From the earlier theorem, $n-\omega_{e}(G) \leq$ $\gamma_{e}^{s n s}(G) \leq n-\omega_{e}(G)+1$. Therefore, $\omega_{e}(G)=\delta_{e}(G)$ or $\delta_{e}(G)+1$. Suppose there exists a $\omega_{e}(G)$ - set $S$ with $|S|=\delta_{e}(G)+1$ such that every vertex in $S$ is equitable adjacent with some vertex in $V-S$. Then, $V-S$ is an equitably strong non-split equitable dominating set of $G$. Therefore, $\gamma_{e}^{\text {sns }}(G) \leq|V-S|=n-\left(\delta_{e}(G)+1\right)$, a contradiction 
to our assumption $\gamma_{e}^{\text {sns }}(G)=n-\delta_{e}(G)$. Therefore, every $\omega_{e}(G)$ - set S with $|S|=\delta_{e}(G)+1$ is such that $S$ contains a vertex not equitably adjacent to any vertex of $V-S$. Conversely, suppose, $\omega_{e}(G)=\delta_{e}(G)+$ 1 and every $\omega_{e}(G)$-set $\mathrm{S}$ with $|S|=\delta_{e}(G)+1$ is such that $S$ contains a vertex not equitably adjacent to any vertex of $V-S$. Therefore, $\mathrm{V}-\mathrm{S}$ is not an equitably strong non-split equitable dominating set of $G$. Therefore, $\gamma_{e}^{\text {sns }}(G)>|V-S|=n-\left(\delta_{e}(G)+1\right)$. That is, $\gamma_{e}^{\text {sns }}(G)>n-\omega_{e}(G)$. But, $\gamma_{e}^{\text {sns }}(G) \leq n-\omega_{e}(G)+1$. Therefore, $\gamma_{e}^{s n s}(G)=n-\omega_{e}(G)+1$.

\section{1. $\gamma_{e}^{\text {sns }}(G)$ for some classes of graphs}

1. $\gamma_{e}^{\text {sns }}\left(K_{n}\right)=1$ where $n \geq 2$.

2. $\gamma_{e}^{\text {sns }}\left(K_{m, n}\right)=m+n-2$, if $|m-n| \leq 1, m, n \geq 2$. Otherwise, $K_{m, n}$ is totally disconnected.

3. $\gamma_{e}^{\text {sns }}\left(C_{n}\right)=n-2$.

4. $\gamma_{e}^{\text {sns }}\left(P_{n}\right)=\{1$, if $n=2$

2 , if $n=3$

$n-3$, if $n \geq 4$.

5. $\gamma_{e}^{\text {sns }}\left(W_{n}\right)=\{1$, if $n=4$

2 , if $n=5$

$n-2$, if $n \geq 6$.

Definition 4. A subset $D$ of $V(G)$ is called an equitable vertex set dominating set of $G$ if for any subset $S$ of $V-D$, there exists a vertex $u \in D$ such that $S \cup\{u\}$ is equitably connected. The minimum cardinality of an equitable vertex set dominating set of $G$ is called the equitable vertex set domination number of $G$ and is denoted by $\gamma_{e}^{v s}(G)$.

Theorem 4. In any graph $G, \gamma_{e}^{v s}(G) \leq \gamma_{e}^{\text {sns }}(G)$.

Proof. Let $D$ be a $\gamma_{e}^{\text {sns }}(G)$-set of $G$. Then, for any subset $\mathrm{S}$ of $V-D,\langle S\rangle$ is equitably complete and for any $v \in S$, there exists a vertex $u \in D$ which is equitably adjacent with $v$. Therefore, $S \cup\{u\}$ is equitably connected. Therefore, $D$ is an equitable vertex set dominating set of $G$ and hence $\gamma_{e}^{v s}(G) \leq|D|=\gamma_{e}^{s n s}(G)$. 


\section{Example 2.}

1. Consider $C_{4}$ with $V\left(C_{4}\right)=\left\{u_{1}, u_{2}, u_{3}, u_{4}\right\}$. Add two vertices $x, y$. Join $\mathrm{x}$ with $u_{1}$ and $u_{4}, \mathrm{y}$ with $u_{2}$ and $u_{3}$ and join $x$ and $y$. Let $G$ be the resulting graph. Then $\left\{u_{1}, u_{4}, x\right\}$ as well as $\left\{u_{2}, u_{3}, y\right\}$ are $\gamma_{e}^{\text {sns }}(G)$-sets of $G$ and hence $\gamma_{e}^{\text {sns }}(G)=3$. The two sets are also $\gamma_{e}^{v s}(G)$ - sets of $G$ and hence $\gamma_{e}^{v s}(G)=3$. Thus, $\gamma_{e}^{v s}(G)=\gamma_{e}^{s n s}(G)=3$.

2. Let $G$ be the graph obtained from $K_{3}$ by attaching a single pendent vertex at a single vertex of $K_{3}$ and joining the pendent vertex with one of the other two vertices of $K_{3}$. Then, the set consisting of the vertex of $K_{3}$ and its pendent vertex is a $\gamma_{e}^{s n s}(G)$ - set of $G$ and hence $\gamma_{e}^{\text {sns }}(G)=2$. The singleton set consisting of that vertex of $K_{3}$ which has a pendent vertex is a $\gamma_{e}^{v s}(G)$ - set of $G$ and hence $\gamma_{e}^{v s}(G)=1<$ $\gamma_{e}^{\text {sns }}(G)$.

Corollary 2. For any equitably connected tree $T, n-\delta_{e}(T) \leq \gamma_{e}^{\text {sns }}(G)$.

Proof. Since $\gamma_{e}^{v s}(G) \leq \gamma_{e}^{\text {sns }}(G)$ and since for any equitably connected tree , $\gamma_{e}^{v s}(G)=n-\delta_{e}(T)$, the result follows.

Theorem 5. If $G$ has no equitable isolate and if $G$ has an equitable strong non-split, equitably independent, equitable dominating set, then $\operatorname{diam}_{e}(G) \leq$ 3.

Proof. Let $G$ be a graph without equitable isolate. Let $D$ be a minimum equitable strong non-split, equitably independent, equitable dominating set. Let $u, v \in V-D$. Then, $d_{e}(u, v)=1$. Let $u \in D, v \in V-D$. Since $G$ has no equitable isolates and $D$ is equitably independent, there exists $w \in V-D$ such that $\mathrm{u}$ and $\mathrm{w}$ are equitably adjacent. Therefore, $d_{e}(u, v) \leq d_{e}(u, w)+d_{e}(w, v)=2$. Let $u, v \in D$. Then there exist $w_{1}, w_{2}$ in $V-D$, such that $u$ and $w_{1}$ are equitably adjacent and $v, w_{2}$ are equitably adjacent. Therefore, $d_{e}(u, v) \leq d_{e}\left(u, w_{1}\right)+d_{e}\left(w_{1}, w_{2}\right)+d_{e}\left(w_{2}, v\right)=3$. Hence $\operatorname{diam}_{e}(G) \leq 3$.

Theorem 6. If $G$ has no equitable isolate and if $\gamma_{e}^{\text {sns }}(G)=\gamma_{e}(G)$, then $\operatorname{diam}_{e}(G) \leq 3$.

Proof. Let $D$ be a $\gamma_{e}^{\text {sns }}(G)$ - set of $G$. Since $D$ is also an equitable dominating set of $\mathrm{G}$ of cardinality $\gamma_{e}(G)$ and since $G$ has no equitable isolate, $V-D$ is an equitable dominating set of $\mathrm{G}$ as well equitably complete set of $G$. Therefore, every vertex of $D$ is equitably adjacent with some vertex of $V-D$. Proceeding as in previous theorem, we get $\operatorname{diam}_{e}(G) \leq 3$. 
Theorem 7. Let $S$ be an equitably independent set in $G$. Let $|D|<n-$ $\Delta_{e}(G)$. Then, $V-D$ is an equitably strong non-split equitable dominating set of $\overline{G_{e}}$, where $\overline{G_{e}}$ is the equitable complement of $G$. (that is, equitable edges of $G$ are to be removed and equitable non-edges are to be added).

Proof. Let $D$ be an equitably independent set in $G$. Let $|D|<n-\Delta_{e}(G)$. Then $|V-D|>\Delta_{e}(G)$. Therefore, each vertex of $D$ is not equitably adjacent with at least one vertex in $V-D$. Therefore, $V-D$ is an equitable dominating set of $\overline{G_{e}}$. Since $D$ is equitably complete in $\overline{G_{e}}, V-D$ is an equitably strong non-split equitable dominating set of $\overline{G_{e}}$.

Definition 5. An equitable dominating set $D$ of an equitably connected graph $G$ is said to be a strong equitably split equitably dominating set of $G$ if $\langle V-D\rangle$ is totally equitably disconnected. The minimum cardinality of such a set is called the strong equitably split equitably domination number of $G$ and is denoted by $\gamma_{e}^{s s}(G)$.

Definition 6. An equitable dominating set $D$ of a graph $G$ is called an equitably regular set dominating set of $G$ if for any subset $S$ of $V-D$, there exists a subset $T$ of $D$ such that $\langle T \cup S\rangle$ is equitably regular. (that is equitable degree of any two vertices of TUS are equal in $\langle T \cup S\rangle$ ). The minimum cardinality of an equitably dominating and equitably regular set dominating set of $G$ is denoted by $\gamma_{e}^{r s}(G)$.

Theorem 8. For any graph $G, \gamma_{e}^{r s}(G) \leq \gamma_{e}^{\text {sns }}(G)+1$.

Proof. Let $D$ be a $\gamma_{e}^{\text {sns }}(G)$ - set of G. Then $\langle V-D\rangle$ is equitably complete. Let $u \in V-D$. Then, $D \cup\{u\}$ is an equitable dominating set of $G$. Also, for any subset $\mathrm{S}$ of $V-D$, there exists $T=\{u\}$ a subset of $D \cup\{u\}$ such that $\langle S \cup T\rangle$ is equitably regular. Hence, $D \cup\{u\}$ is an equitable dominating and equitably regular set dominating set of $\mathrm{G}$. Therefore, $\gamma_{e}^{r s}(G) \leq|D \cup\{u\}|=$ $\gamma_{e}^{\text {sns }}(G)+1$.

Example 3. In the Example 2(1) of Theorem 2.4, $\gamma_{e}^{r s}(G)=4$ and $\gamma_{e}^{\text {sns }}(G)=$ 3 and in Example 2(2) of Theorem 2.4, $\gamma_{e}^{r s}(G)=\gamma_{e}^{\text {sns }}(G)=2$.

Theorem 9. Let $G$ be a graph without equitable isolate. If $\operatorname{diam}_{e}(G) \leq 3$, then $\gamma_{e}^{\text {sns }}(G) \leq n-t$ where $t$ is the number of equitable cut vertices of $G$.

Proof. Let $T$ be the set of all equitable cut vertices. Then $|T|=t$. If $t=0$ or 1 , then the result is obviously true. Let $t \geq 2$. Let $u$ and $v$ belong 
to $T$. If $u$ and $v$ are equitably non-adjacent, then there exist an equitable component say $V_{1}$ containing $v$ in $V-\{u\}$. Since $u$ and $v$ are equitable nonisolates, there exists a vertex $u_{1}$ equitably adjacent with $\mathrm{u}$ in an equitable component say $V_{2}$ different from $V_{1}$. Also, there exists a vertex $v_{1}$ in $V_{1}$ equitably adjacent with $v$. Thus, $d_{e}\left(u_{1}, v_{1}\right) \geq 4$, a contradiction since $\operatorname{diam}_{e}(G) \leq 3$. Therefore, any two vertices of $T$ are equitably adjacent. Also, every vertex in $T$ is equitably adjacent with atleast one vertex in $V-T$. Therefore, $V-T$ is a equitably strong non-split equitable dominating set of $\mathrm{G}$. That is, $\gamma_{e}^{\text {sns }}(G) \leq|V-T|=n-t$.

Definition 7. An equitable dominating set $D$ of a graph $G$ is called an equitably non-split equitable dominating set if $\langle V-D\rangle$ is equitably connected. The minimum cardinality of such a set is called the equitably non-split equitable dominating number of $G$ and is denoted by $\gamma_{e}^{\text {ns }}(G)$.

Theorem 10. Let $G$ be a simple graph without equitable isolate. Also, every vertex of $G$ is either an equitable cut vertex or a vertex of equitable degree one. If $\omega_{e}(G)=k$ then, $\gamma_{e}^{n s}(G)=\gamma_{e}^{\text {sns }}(G)=n-k$.

Proof. Let $S$ be the set of all equitable cut vertices with $|S|=k$. From the above theorem we get, $S$ is equitably complete. Also, every vertex in $S$ is equitably adjacent with a vertex of equitable degree one. Therefore, $V-S$ is an equitably non-split equitable dominating set as well as equitably strong non-split equitable dominating set of $G$. $|V-S|=n-k$. If $\gamma_{e}^{\text {sns }}(G)<n-k$, then the complement of a $\gamma_{e}^{\text {sns }}(G)$ - set will contain a least $k+1$ elements which form an induced equitably complete subgraph, a contradiction since $\omega_{e}(G)=k$. Therefore, $\gamma_{e}^{\text {sns }}(G)=n-k$. Also, if $\gamma_{e}^{n s}(G)<n-k$, then the complement of a $\gamma_{e}^{n s}(G)$ - set say $D$ will contain a least $k+1$ elements in the complement and hence at least one vertex of equitable degree one which can be dominated only by an equitable cut vertex. Therefore, $D$ contains an equitable cut vertex which means that $\langle V-D\rangle$ is equitably disconnected, a contradiction since $D$ is a $\gamma_{e}^{n s}(G)$ - set of $G$. Therefore, $\gamma_{e}^{n s}(G)=n-k$. Hence the theorem.

Definition 8. A subset $D$ of $V(G)$ of a simple graph is called an equitably efficient dominating set of $G$ if every vertex of $V-D$ is equitably dominated by exactly one vertex of $D$. The minimum cardinality of such a set is called the equitably efficient domination number of $G$ and is denoted by regular $\gamma_{e}^{e}(G)$. 
Theorem 11. Let $G$ be a equitably $n$ - regular (that is, the equitable degree of every vertex is $n$ ) graph of order $2 n$. If $D$ is an equitably efficient equitably dominating set of $G$, then $D$ and $V-D$ are equitably strong non-split equitable dominating sets of $G$.

Proof. Let $G$ be a simple graph which is equitably $n$ - regular and of order $2 n$. Let $D$ be an equitably efficient equitably dominating set of $G$, Then, any vertex of $V-D$ is equitably adjacent with exactly one vertex of $D$. Therefore, the remaining $n-1$ equitable neighbours of that vertex are in $V-D$. This is true for any vertex of $V-D$. Therefore, $V-D$ is equitably complete and $|V-D|=n$. Therefore, $|D|=n$. Since $G$ is equitably $n$ - regular and $|D|=n$, any vertex of $D$ is adjacent equitably with at least one vertex of $V-D$. Suppose, there exists a vertex $u \in D$ which is adjacent equitably with two or more vertices of $V-D$. Also, since $\operatorname{deg}_{e}(u)=n$, and $|D|=n$, there exists a vertex $v \in D, v \neq u$ such that $u$ and $v$ are not equitably adjacent. Also, $v$ can not be equitably adjacent with any equitable neighbour of $u \in V-D$. Proceeding in this way since $|V-D|=n$, we reach a stage when there exists a vertex in $D$ whose equitable degree is $<n$, a contradiction. Therefore, every vertex of $D$ is equitably adjacent with exactly one vertex in $V-D$ and $\langle D\rangle$ is equitably complete. Hence $D$ and $V-D$ are equitably strong non-split equitable dominating sets of $G$.

Theorem 12. Let $G$ be a simple graph without equitable isolates and with $\Delta_{e}(G) \leq n-2$. Let $D$ be an equitably strong non-split equitable dominating set of $G$ such that $\langle D\rangle$ is equitably complete and $|D| \leq \delta_{e}(G)$. Then $D$ is minimal and $V-D$ is also a minimal equitably strong non-split equitable dominating set of $G$.

Proof. Suppose, there exists a vertex in $D$ which is equitably adjacent with every vertex of $V-D$. Then $G$ is equitably complete. That is, $\Delta_{e}(G)=n-1$, a contradiction. Therefore, every vertex in $D$ is not adjacent with some vertex of $V-D$. Therefore, $D$ is minimal.

If $|D| \leq \delta_{e}(G)$, then every vertex in $D$ is equitably adjacent with some vertex of $V-D$. Therefore, $V-D$ is an equitably strong non-split equitable dominating set of $G$. Further, arguing as above, each vertex in $V-D$ is not equitably adjacent with some vertex of $D$. Therefore, $V-D$ is also a minimal equitably strong non-split equitable dominating set of $G$.

Theorem 13. Let $G$ be a simple graph without equitable isolate. If $\Delta_{e}(G)<$ $\alpha_{e}(G)$, then $\gamma_{e}^{\text {sns }}(\bar{G})=n-\omega_{e}(\bar{G})$. 
Proof. Let $T$ be a minimum equitable vertex cover of $G$ so that $|T|=\alpha_{e}(G)$. If $G=K_{n}$, then $\beta_{e}(G)=1, \alpha_{e}(G)=n-1=\Delta_{e}(G)$. But $\Delta_{e}(G)<\alpha_{e}(G)$. Therefore, $G=K_{n} . V-T$ is equitable independent. Since $|V-T|=$ $\beta_{e}(G)$ and $G=K_{n},|V-T| \geq 2$. If a vertex say $u \in V-T$ is equitably adjacent with every vertex of $\mathrm{T}$, then $\operatorname{deg}_{e}(u) \geq|T|=\alpha_{e}(G)>\Delta_{e}(G)$, a contradiction. Therefore, every vertex of $\mathrm{V}-\mathrm{T}$ is not adjacent with at least one vertex of $T$. Therefore, $\mathrm{T}$ is an equitably strong non-split equitable dominating set of $\bar{G}$. Hence, $\gamma_{e}^{\text {sns }}(\bar{G}) \leq|T|=\alpha_{e}(G)=n-\beta_{e}(G) \leq$ $n-\omega_{e}(\bar{G})$. But, $\gamma_{e}^{\text {sns }}(\bar{G}) \geq n-\omega_{e}(\bar{G})$. Hence, $\gamma_{e}^{\text {sns }}(\bar{G})=n-\omega_{e}(\bar{G})$.

Theorem 14. Let $G$ be a graph without equitable isolate. Let $G$ and $\bar{G}$ be equitably connected. Further, let $\omega_{e}(G) \geq \delta_{e}(G)$ and $\omega_{e}(\bar{G}) \geq \delta_{e}(\bar{G})$. Then, $\gamma_{e}^{\text {sns }}(G)+\gamma_{e}^{\text {sns }}(\bar{G}) \leq n+1+\Delta_{e}(G)-\delta_{e}(G)$.

Proof. From Theorem 2.3, $\gamma_{e}^{\text {sns }}(G) \leq n-\delta_{e}(G)$ and $\gamma_{e}^{\text {sns }}(\bar{G}) \leq n-\delta_{e}(\bar{G}) \leq$ $1+\Delta_{e}(G)$. Hence the theorem.

Remark 4. Let $G=C_{5}$. Then $\bar{G}=C_{5} \cdot \gamma_{e}^{\text {sns }}(G)=3=\gamma_{e}^{\text {sns }}(\bar{G}) . \delta_{e}(G)=$ $\delta_{e}(G)=2 . n=5$. Therefore, $\gamma_{e}^{\text {sns }}(G)+\gamma_{e}^{\text {sns }}(\bar{G})=6, n+1+\Delta_{e}(G)-\delta_{e}(G)=$ $5+1+2-2=6$. Hence equality holds in the above theorem when $G=C_{5}$.

\section{References}

[1] K. D. Dharmalingam, "Studies in graph theorey-equitable domination and Bottleneck domination", Ph.D. Thesis, Madurai Kamaraj University, Madurai, 2006.

[2] K. M. Dharmalingam, "A note on the equitable covering and equitable packing of a graph", Bulletin of international mathematical virtual institute, vol. 3, pp. 21-27, 2013. [Online]. Available: https:// bit.ly/ 3y8D5HQ

[3] K. M. Dharmalingam, "Equitable associate graph of a graph", Bulletin of international mathematical virtual institute, vol. 2, pp. 109-116, 2012. [Online]. Available: https:/ / bit.ly/ 2VfpboM

[4] V. R. Kulli and B. Janakiram, "The strong non-split domination number of a graph", International journal of management and systems, vol. 19, no. 2, pp. 145-156, 2003. [Online]. Available: https:/ / bit.ly/ 3y5ciMt 
[5] V. R. Kulli and B. Janakiram, "The split domination number of a graph", Graph theory notes of New York, vol. 32, pp. 16-19. [Online]. Available: https:/ / bit.ly/ 3zN6VlF

[6] W. Meyer, "Equitable coloring", The American mathematical monthly, vol. 80, No. 8, pp. 920-922, 1973, doi: 10.2307/2319405

[7] V. Swaminathan and K. M. Dharmalingam, "Degree equitable domination in graphs", Kragujevac journal of mathematics, vol. 35, no. 1, pp. 191-197, 2011. [Online]. Available: https:// bit.ly/ 3i3aLB5 